\title{
Decreased expression of KPNA2 in Hepatocellular carcinoma infers favorable prognosis and high immune infiltrating level
}

\author{
Kang-Ming Zhu \\ Shandong University \\ Hui Yuan \\ Shandong University \\ Jing Li ( $\nabla_{\text {jingli1984@email.sdu.edu.cn ) }}$ \\ Zibo Central Hospital, Binzhou Medical University
}

\section{Research Article}

Keywords: Hepatocellular carcinoma, Biomarker, Immune infiltration, KPNA2, Prognosis, Lymphocyte

Posted Date: March 1st, 2022

DOI: https://doi.org/10.21203/rs.3.rs-691618/v2

License: (c) (i) This work is licensed under a Creative Commons Attribution 4.0 International License.

Read Full License 


\section{Abstract}

\section{Background}

Liver hepatocellular carcinoma (LIHC) is an important pathological type of liver cancer. The immune infiltration of the tumor microenvironment is negatively correlated with the overall survival rate of LIHC. At present, the role and molecular mechanism of KPNA2 in LIHC have not been elucidated, and the prognostic correlation between the two and the immune infiltration of $\mathrm{LIHC}$ are still unclear. Our study evaluated the role of KPNA2 in LIHC through TCGA data.

\section{Method}

Gene expression profiling interactive analysis (GEPIA) is used to analyze the expression of KPNA2 in LIHC. We evaluated the impact of KPNA2 on the survival of LIHC patients through the survival module. Then, We downloaded the LIHC data set from TCGA. Logistic regression was used to analyze the correlation between clinical information and KPNA2 expression. Cox regression analysis was used to analyze the clinicopathological characteristics related to the overall survival rate of TCGA patients. In addition, we used the "correlation" modules of CIBERSORT and GEPIA to explore the correlation between KPNA2 and cancer immune infiltrate. Western blotting was used to detect the expression of KPNA2.

\section{Result}

Using logistic regression for univariate analysis, increased KPNA2 expression was significantly correlated with pathological stage, tumor status, and lymph node status. In addition, multivariate analysis showed that down-regulation of KPNA2 expression, negative pathological stage and distant metastasis are independent prognostic factors for good prognosis. Specifically, CIBERSORT analysis was used to establish a negative correlation between the up-regulated expression of KPNA2 and the level of immune infiltration of B cells, NK cells, mast cells, and T cells. In addition, we confirmed this in the "Association" module of GEPIA. The expression of KPNA2 in LIHC tissues was significantly lower than that in adjacent normal tissues by western blotting.

\section{Conclusion}

The down-regulation of KPNA2 expression is associated with a good prognosis and an increase in the proportion of immune cells in LIHC. These conclusions indicate that KPNA2 is related to the level of immune infiltration of LIHC and can be used as a potential prognostic biomarker of LIHC and a potential target for clinical tumor treatment.

\section{Introduction}

Primary liver cancer, a common clinical malignant tumor, accounts for the second leading cause of cancer-related death in the world [1], and the 5-year overall survival rate is only 18\% [2], among which Liver hepatocellular carcinoma (LIHC) is mainly. Surgical resection is the main treatment method in 
clinical practice, but the surgical treatment effect and prognosis are poor. Data shows that the recurrence rate within 5 years after surgery is more than $70 \%$ [3]. Therefore, finding new markers of hepatocellular carcinoma is of great significance for the treatment of hepatocellular carcinoma.

KPNA2 protein is a nuclear transport protein that contains $\mathrm{N}$-terminal, C-terminal and repeated ARM sequences. It binds to nuclear proteins, enters the nucleus to play a role in protein transport, participates in the transport of a variety of oncogenic proteins, and regulates a variety of cellular activities [4]. The high expression of nuclear transporter gene 2 (karyopherin alpha 2, KPNA2) has also been confirmed to be associated with a variety of malignant tumors such as rectal cancer [5], breast cancer [6], ovarian cancer [7], lung adenocarcinoma [8], etc. It is closely linked with tumor progression and prognosis. But the role and molecular mechanism of KPNA2 in LIHC have not yet been elucidated, and the prognostic correlation between the two and the immune infiltration of LIHC are still unclear.

This study will use gene expression analysis interaction analysis (GEPIA), COX regression analysis and CIBERSORT algorithm to analyze the relevant data in the TCGA database, and explore the correlation between KPNA2 and LIHC prognostic value and immune infiltration, so as to predict its possibility The molecular mechanism provides a new molecular target for LIHC.

\section{Materials And Methods}

\section{Data collection}

The LIHC patient data sets with gene expression profiles and paired clinical information used in this study are all from The Cancer Genome Atlas (TCGA), with 424 samples in total, including 374 carcinoma tissues and 50 adjacent normal tissues. Subsequently, cases with insufficient or missing data on age, overall survival time, and TNM staging were excluded. Finally, the clinical data of 343 eligible patients were retained for Cox regression analysis. In order to study the influence of the immune microenvironment on the expression of KPNA2, we used 374 tumor tissues for CIBERSORT analysis.

To

\section{GEPIA Survival and Expression Analysis}

Gene Expression Analysis Interactive Analysis (GEPIA) is a web-based online gene expression analysis database used to evaluate the correlation between KPNA2 expression and clinicopathological information of liver cancer Sex. GEPIA is a web server[9] that uses standard processing pipelines to analyze the RNA sequencing expression data of 9736 tumors and 8587 normal samples, which come from the TCGA and GTEx projects. GEPIA can analyze the correlation between the expression level of KPNA2 and the prognosis of LIHC. At the same time, with the disease state (tumor or normal) as a variable, a box plot is drawn dynamically to visualize the differential expression of KPNA2. Analyze the clinical stage diagram with pathological stage as the variable, and compare the expression level of KPNA2 in different pathological stages. 


\section{Evaluation of tumor infiltrating immune cells}

CIBERSORT is a deconvolution algorithm based on gene expression, which can evaluate the expression changes of a group of genes relative to all other genes in the sample. Therefore, the TIIC concentration can be accurately estimated through this process. The stable performance of CIBERSORT encourages people to pay more and more attention to cell heterogeneity research [10], [11], [12]. Our current analysis uses CIBERSORT to measure the proportion of $22 \mathrm{TIICs}$ in LIHC to assess its correlation with survival rates and molecular subpopulations. In short, gene expression datasets are listed using standard annotation files and uploaded to the CIBERSORT web portal, and the algorithm runs with its default signature matrix in one thousand permutations. CIBERSORT estimates the P value of deconvolution through Monte Carlo sampling, thereby establishing a measure of confidence in the result. To evaluate the influence of KPNA2 expression, we used 424 samples in TCGA, which represent the complete gene range. The $p$ value $<0.05$ was used as the criterion for selecting lymphocytes that may be affected by KPNA2 expression. In order to detect the correlation between 22 kinds of immune cells, we drew a correlation heat map.

In addition, the "correlation" module of GEPIA was used to further confirm the relationship between the expression of KPNA2 and possible tumor infiltrating immune cell gene markers. Gene markers involve T cells (general), CD8 + T cells, B cells, neutrophils, natural killer cells (NK), T helper 1 (Th1) cells, T helper 2 (Th2) cells, follicular helper T (Tfh) cells, T helper 17 (Th17) cells, exhausted T cells and mast cells. Existing studies have provided references for [13], [14], and [15] gene markers. Related modules help to draw custom expression scatter plots of gene pairs for specific cancer types, as well as Spearman's R and predicted statistical significance. The $P$ value $<0.01$ is determined as the threshold value. In order to detect the correlation between 22 kinds of immune cells, we drew a correlation heat map, that is, a correlation chart between every two different kinds of immune cells in the sample.

\section{Western blotting}

Western blotting is a protein detection technique that transfers the total protein of cells or tissues separated by electrophoresis from the gel to the solid support NC membrane or PVDF membrane, and then uses a specific antibody to detect a specific antigen. It is now widely used Research on gene expression at the protein level, detection of antibody activity, and early diagnosis of diseases. In this study, western blotting was used to detect the expression of KPNA2 in liver cancer tissues and adjacent normal tissues.

\section{Statistical Analysis}

The statistical data obtained by TCGA is realized by R-3.5.3. Logistic regression was used to analyze the correlation between clinical characteristics and KPNA2 expression. Perform COX regression analysis to determine the overall survival-related clinical characteristics of TCGA patients. In this study, $\mathrm{p}$ value $<0.05$ was used as the threshold for statistically significant differences. The correlation of gene expression was 
judged by Spearman's $R$ and statistical significance. The absolute value of $R$ greater than 0.1 is considered relevant, and the value of $\mathrm{P}<0.01$ is considered statistically significant.

\section{Results}

\subsection{Survival outcomes and multivariate analysis}

As shown in Fig. 1, increased expression of KPNA2 is significantly correlated with poor overall survival (Fig. 1A, $P<0.001$ ) and advanced pathological stage (Fig. 1B, $P<0.001$ ). In addition, KPNA2 expression in tumor samples is obviously lower than in normal (Fig. 1C). As shown in Table 1a, Univariate analysis using Cox regression revealed that in different tumor states, the influencing factors have different effects on the overall survival rate. In tumor stage $(I-I)$, age $(H R=1.023, P<0.05)$ the expression of KPNA2 $(H R=$ $1.705, \mathrm{P}=0.000$ ) are significantly associated with overall survival. In tumor stage (III-IV), the expression of KPNA2 (HR $=1.646, P=0.001)$ are significantly associated with overall survival. In multivariate analysis (Fig. 2), the down-regulated KPNA2 expression is independent prognostic factor of favorable prognosis.

\subsection{Association between KPNA2 expression and clinicopathologic variables}

The underlying mechanism of KPNA2 expression in cancer requires further study, hence we analyzed and correlated it with certain clinical aspects in cases of liver carcinoma. Cases with eligible clinical information were analyzed by R-3.5.3. As shown in Table 2, univariate analysis using logistic regression with KPNA2 expression as a categorical dependent variable revealed that increased expression of KPNA2 correlated significantly with the pathological stage (I vs II, $p=0.007$; III vs I, $p=0.002$ ), tumor status (T1 vs T2, $p=0.012$; T1 vs T3, $p=0.006$; T1-T2 vs T3-T4, $p=0.033$ ), and grade (II vs III, $p=0.011$; I vs III, $p=$ 0.028 ; I-II vs IIII-IV, $p=0.001$ ).

\subsection{Relationship between KPNA2 expression and tumor-infiltrating immune cells}

Previous analyses suggest tumor-infiltrating lymphocytes as independent predictors of sentinel lymph node status and survival in cancer patients [16]. Therefore, we tried to find whether KPNA2 expression relates to immune infiltration in liver carcinoma. Among 374 liver carcinoma samples, samples with the top $1 / 3$ and the lowest 1/3 KPNA2 expression were included into high expression group and low expression group, respectively. We use an established computational resource (CIBERSORT) to explore gene expression profiles of downloaded samples to infer the ratio of 22 types of immune cells in high and low KPNA2 expression groups. The results of CIBERSORT were exhibited in Fig. 3 . The proportions of 22 subpopulations of immune cells were clearly rendered on it. As shown in Fig. 3A, main immune cells including $B$ cells naive, $B$ cells memory, $T$ cells CD4 memory resting, T cells CD 4 memory activated, $T$ cells follicular helper, $T$ cells regulatory (Tregs), $T$ cells gamma delta, NK cells resting, monocytes, macrophages $\mathrm{M} 0$, macrophages $\mathrm{M} 1$, dendritic cells resting, mast cells resting, and neutrophils were affected by KPNA2 expression. Among them, B cells memory $(p=0.002)$, T cells CD4 memory activated $(p<0.001)$, T cells follicular helper $(p<0.001)$, Macrophages M0 $(p<0.001)$, Dendritic cells resting $(p=$ $0.031)$, Neutrophils $(p=0.036)$ share a higher proportion in high expression group compared with low expression group. In contrast, the proportion of B cells naïve $(p=0.002)$, T cells CD4 memory resting $(p=$ 
$0.002)$, NK cells resting $(p=0.023)$, Monocytes $(p=0.005)$, macrophages M1 $(p=0.002)$, Mast cells resting ( $p=0.001)$, are apparently lower. In addition, correlation heatmap (Fig. 3B) revealed that the proportions of different TIICs subpopulations were weakly to moderately correlated.

"Correlation" module of GEPIA helped us to analyze the link between KPNA2 expression and gene markers of different types of tumor-infiltrating immune cells, including CD $8+T$ cells, $T$ cells (general), B cells, neutrophils, Mast cells and DCs, as also different functional T cells, namely Th1, Th2, Tfh, Th17, Tregs, and exhausted T cells (Table 3). Results confirmed that KPNA2 expression is correlated with many marker sets of different immune cells in liver carcinoma. The gene markers effected by KPNA2 expression include CD8A, CD8B of CD8 + T cell, CD2, CD3E of T cell (general), KIR2DL3, KIR2DL4 of Natural killer cell, GATA3, STAT5A of Th2, STAT3 of Th17, CTLA4, LAG3 of T cell exhaustion, as well as HDC of Mast cells. Correlations were evaluated using Spearman correlation coefficient. Correlation results between KPNA2 and markers of B cells, Mast cells and T cells were similar to CIBERSORT. Thus, these findings indicate that KPNA2 may play a crucial role in regulating the abundance of $B$ cells, Mast cells and $T$ cells. Further studies need to be done to explore whether KPNA2 is a significant factor that relate to immune infiltration of Neutrophils.

Western blotting (Fig. 4) results showed that the expression of KPNA2 in liver cancer tissues was significantly lower than that in normal tissues adjacent to cancer.

\section{Discussion}

In previous studies, high KPNA2 levels were associated with poor prognosis and high recurrence rate of breast cancer [17]. In many studies, KPNA2 is associated with many other types of malignant tumors, including the endometrium, prostate, and brain. Here, we found that changes in the level of KPNA2 expression are correlated with the prognosis of LIHC. Down-regulation of KPNA2 expression is an independent prognostic factor for good prognosis. At the same time, in the context of clinical data, we conducted a correlation analysis of KPNA2. The increase of KPNA2 expression level is significantly related to pathological staging, tumor status, lymph node status and other clinical characteristics. Therefore, the results of previous studies indicate that KPNA2 may have a potential impact on tumor immunology and can be used as a promising cancer biomarker.

In this study, we used the online database GEPIA to find the correlation between the expression of KPNA2 and the survival rate of $\mathrm{LIHC}$ patients. The down-regulation of KPNA2 expression is related to a good prognosis. We also observed that the expression of KPNA2 is different in normal tissues and tumor tissues of LIHC. In order to further study the underlying mechanism of KPNA2 expression in cancer and its relationship, we downloaded the data set from TCGA. The TCGA analysis of R-3.5.3 showed that the expression of KPNA2 was related to the pathological stage, tumor status, lymph node status and other clinical features. Multivariate analysis showed that KPNA2 expression is an independent prognostic factor for the prognosis of LIHC patients. 
In our study, an important conclusion relates to the correlation between the expression of KPNA2 and the level of LIHC immune infiltration. CIBERSORT analysis showed that the expression of KPNA2 in LIHC was significantly positively correlated with the level of T cell and NK cell infiltration. Similarly, the relationship between different immune cells' gene markers and the expression of KPNA2 also suggests the importance of KPNA2 in regulating the immune microenvironment of LIHC tumors. First, using the CIBERSORT algorithm, it was found that the ratios of B cells, several T cells, NK cells, monocytes, macrophages and mast cells in the KPNA2 high expression group were distinctly lower than that in the low expression group. Additionally, we used the "association" module of GEPIA to confirm that KIR2DL3 and KIR2DL4 of NK cells are negatively correlated with the expression of KPNA2. This result shows us the potential regulatory role of KPNA2 in a large number of tumor-associated NK cells. CD8 + T cell, T cell (general), Th2, Th17, T cell exhaustion are different functional T cells. The increase of KPNA2 expression is negatively correlated with its markers such as CD8A, CD8B, CD2, CD3E, GATA3, STAT5A, STAT3, CTLA4, LAG3. These correlations may indicate a possible mechanism by which KPNA2 regulates $T$ cell function in LIHC. The low expression of T cell and NK cell signal is related to the prolonged overall survival rate, which can be predicted in many types of tumors including liver cancer [18]. Together, these findings indicate that KPNA2 plays a key role in regulating and recruiting immune infiltrating cells in LIHC.

The analysis of the role of TIICs (tumor infiltrating immune cells) in human tumors usually focuses on T cells, some of which report their response to immune checkpoint suppression and their survival rate [19], [20]. This study complements the evolving literature that identifies T cells as a positive prognosis [21]. More and more evidences show that hypoxic stress in liver cancer tissues, the conversion of activating/inhibitory NK receptors (NKR), and the influence of immunomodulatory components in TME, to a large extent, lead to abnormal NK cell function. Significantly related to fatigue, anti-tumor immunity and poor prognosis [22], [23]. More and more evidences show that the intrahepatic immune response involves different lymphocyte populations, and their contributions to HCC immune surveillance are different. The intratumoral balance of regulating $T$ cells and cytotoxic $T$ cells plays a meaningful role in assessing the immune status and progression of liver cancer [24], [25]. Therefore, the positive effect of KPNA2 on LIHC may be consistent with the lower density of $T$ cells and B cells, which suggests a possible mechanism for KPNA2 to affect the overall survival of LIHC.

KPN 31 is an important paralog of KPNA2, and the tumorigenesis mechanism of KPN 31 activation is still unclear. Nuclear transporters play an important role in regulating the development of malignant tumor. According to reports, the nuclear import protein $\mathrm{Kpn} \beta 1$ is an anti-tumor target for cervical cancer [26]. KPN $\beta 1$ has recently been shown to regulate the proliferation of human glioma cells via the Wnt- $\beta$-catenin pathway. [27] In addition, Yang et al. [28] showed that the expression of $\mathrm{Kpn} \beta 1$ is up-regulated in liver cancer, and Kpn $\beta 1$ may become a contributing factor of liver cancer through the NF-KB signaling pathway and serve as an independent prognostic indicator and a new target in the treatment strategy for liver cancer. Although there is no detailed explanation of the role of KPNA2 in LIHC, based on our findings and previous studies on KPN $\beta$ 1, we have reason to believe that the role of KPNA2 may be a potential mechanism for LIHC to down-regulate the level of immune infiltration. 
In conclusion, down-regulated KPNA2 expression is associated with a good prognosis. At the same time, the altered expression of KPNA2 is associated with different ratios of immune cells such as NK cells and T cells in LIHC. Therefore, the immune infiltration mechanism of KPNA2 in the progression of LIHC has important clinical significance. It may become a possible prognostic biomarker of $\mathrm{LIHC}$, and it can be used for gene-targeted therapy in tumor tissues with high KPNA2 expression.

\section{Abbreviations}

Hepatocellular carcinoma (LIHC)

Gene expression profiling interactive analysis (GEPIA)

The Cancer Genome Atlas (TCGA)

natural killer cells (NK)

T helper 1 cells (Th1)

T helper 2 cells (Th2)

follicular helper T cells ( Tfh)

T helper 17 cells (Th17)

T cells regulatory (Tregs)

\section{Declarations}

Ethics approval and consent to participate

Not applicable

Consent for publication

Not applicable

Availability of data and material

The datasets used and/or analysed during the current study are available from the corresponding author on reasonable request.

Competing interests

The authors declare that they have no competing interests

Funding 
Not applicable

Authors' contributions

Kang-Ming Zhu contributed significantly to analysis and manuscript writing;

Yun-Di Zhang involved in scientific design and performed the data analyses;

Hui Yuan was involved in manuscript writing;

All authors contributed to drafts of the manuscript and approved the final version.

Acknowledgements

Not applicable

\section{References}

1. Mittal Sahil and El-Serag Hashem B. Epidemiology of hepatocellular carcinoma: consider the population.[J]. Journal of clinical gastroenterology, 2013, 47 Suppl : S2-6.

2. Duan Yiping,Chen Liangyue,Liu Jiacui,Huang Ben,Cheng Qingyuan,Ma Tiantian,Zhu Cuiwen,Qin Fei.Analysis of the clinical significance and molecular mechanism of PFKFB4 in hepatocellular carcinoma based on TCGA database[J].Journal of Molecular Diagnosis and Therapy,2021,13(01):2529.

3. Shi Junying, Wang Ye, Chen Wen, et al. Experimental study of GeXP multiplex analysis technology for detection of long-chain non-coding RNA expression in liver cancer tissues[J].Journal of Molecular Diagnosis and Therapy $₫ 2018,10(1): 9-16$.

4. Zhou Ying, Han Xu.Research progress on the relationship between KPNA2 and epithelial ovarian cancer[J].Medical Review,2020,26(16):3216-3220.

5. Huang $B$ and Luo $H$ and Guo X B. High expression of KPNA2 promotes colorectal cancer development by activating the PI3K/AKT pathway.[J]. Journal of biological regulators and homeostatic agents, 2020, 34(6)

6. Xue Meiling,Zhang Xue,Duan Xiuqing.The role of nuclear transporter gene 2 in the occurrence and development of breast cancer[J].Medical Review,2020,26(14):2775-2779.

7. Zhou Ying, Han Xu.Research progress on the relationship between KPNA2 and epithelial ovarian cancer[J].Medical Review,2020,26(16):3216-3220.

8. Niu Yue,Shi Lei,Zhang Ting,Sun Xinxin,Bai Jianping.Expression of trophophilic virus integration site 1 and nuclear transporter gene 2 in triple-negative breast cancer tissues and its relationship with clinicopathological features and prognosis[J].China Journal of Modern Medicine,2021,31(05):8-14.

9. Z. Tang, C. Li, B. Kang, G. Gao, C. Li, Z. Zhang, GEPIA: a web server for cancer and normal gene expression profiling and interactive analyses, Nucl. Acids Res. 45 (Web Server issue) (2017). 
10. H.R. Ali, L. Chlon, P.D. Pharoah, F. Markowetz, C. Caldas, Patterns of immune in-filtration in breast cancer and their clinical implications: a gene-expression-based retrospective study, PLoS Med. 13 (12) (2016) e1002194.

11. X. Liu, S. Wu, Y. Yang, M. Zhao, G. Zhu, Z. Hou, The prognostic landscape of tumor- infiltrating immune cell and immunomodulators in lung cancer, Biomed. Pharmacother. 95 (2017) 55-61.

12. R.D. Bense, C. Sotiriou, M.J. Piccartgebhart, et al., Relevance of tumor-infiltrating immune cell composition and functionality for disease outcome in breast cancer, J. Natl. Cancer Inst. 109 (1) (2016) djw192.

13. N.O. Siemers, J.L. Holloway, H. Chang, Genome-wide association analysis identifies genetic correlates of immune infiltrates in solid tumors, PLoS ONE 12 (7) (2017) e0179726.

14. P. Danaher, S. Warren, L. Dennis, et al., Gene expression markers of tumor in-filtrating leukocytes, J. ImmunoTher. Cancer 5 (1) (2017) 18.

15. S. Sousa, J. Määttä, The role of tumour-associated macrophages in bone metastasis, Bone Oncol. 5 (3) (2016) 135-138.

16. F. Azimi, R.A. Scolyer, P. Rumcheva, et al., Tumor-infiltrating lymphocyte grade is an independent predictor of sentinel lymph node status and survival in patients with cutaneous melanoma, J. Clin. Oncol. 30 (21) (2012) 2678-2683.

17. Dahl E, Kristiansen G, Gottlob K, Klaman I, Ebner E, Hinzmann B, Hermann K, Pilarsky C, Durst M, Klinkhammer-Schalke $\mathrm{M}$, et al. Molecular profiling of laser-microdissected matched tumor and normal breast tissue identifies karyopherin alpha2 as a potential novel prognostic marker in breast cancer. Clin Cancer Res. 2006;12(13):3950-3960. doi: 10.1158/1078-0432.CCR-05-2090. [PubMed] [CrossRef] [Google Scholar]

18. M.D. Iglesia, J.S. Parker, K.A. Hoadley, J.S. Serody, C.M. Perou, B.G. Vincent, Genomic analysis of immune cell infiltrates across 11 tumor types, J. Natl. Cancer Inst. 108 (11) (2016) djw144.

19. W. Zhu, C. Germain, Z. Liu, et al., A high density of tertiary lymphoid structure B cells in lung tumors is associated with increased CD4+ T cell receptor repertoire clonality, Oncoimmunology 4 (12) (2015).

20. D. Huangfu, A. Liu, A.S. Rakeman, N.S. Murcia, L. Niswander, K.V. Anderson, Hedgehog signalling in the mouse requires intraflagellar transport proteins, Nature 426 (6962) (2003) 83-87

21. P. Tran, C. Haycraft, T. Besschetnova, et al., THM1 negatively modulates mouse sonic hedgehog signal transduction and affects retrograde intraflagellar transport in cilia, Nat. Genet. 40 (4) (2008) 403-410.

22. Kamimura H, et al. Reduced NKG2D ligand expression in hepatocellular carcinoma correlates with early recurrence. J Hepatol. 2012;56(2):381-388. doi: 10.1016/j.jhep.2011.06.017.

23. Hasmim $M$, et al. Critical role of tumor microenvironment in shaping NK cell functions: implication of hypoxic stress. Front Immunol. 2015;6:482. doi: 10.3389/fimmu.2015.00482.

24. . Gao Q, et al. Intratumoral balance of regulatory and cytotoxic T cells is associated with prognosis of hepatocellular carcinoma after resection. J Clin Oncol. 2007;25(18):2586-2593. doi: 10.1200/JCO.2006.09.4565. 
25. . Prieto J, Melero I, Sangro B. Immunological landscape and immunotherapy of hepatocellular carcinoma. Nat Rev Gastroenterol Hepatol. 2015;12(12):681-700. doi: 10.1038/nrgastro.2015.173.

26. Angus $L$, van der Watt PJ, Leaner VD. Inhibition of the nuclear transporter, Kpnbeta1, results in prolonged mitotic arrest and activation of the intrinsic apoptotic pathway in cervical cancer cells. Carcinogenesis 2014, 35: 1121-1131.

27. Lu T, Bao Z, Wang Y, et al. Karyopherin- $\beta 1$ regulates proliferation of human glioma cells via Wnt/ $\beta$ catenin pathway. Biochem Biophys Res Commun. 2016;478(3):1189-1197.

28. Yang L, Hu B, Zhang Y, Qiang S, Cai J, Huang W, Gong C, et al. Suppression of the nuclear transporter-KPNbeta1 expression inhibits tumor proliferation in hepatocellular carcinoma. Med Oncol 2015, 32: 128.

\section{Tables}

Table1

Clinicopathologic variable HR HR.95L HR.95H pvalue Tumor stage(III).

\begin{tabular}{lllll}
\hline age & 1.023 & 1.002 & 1.045 & 0.029 \\
\hline gender & 0.634 & 0.387 & 1.039 & 0.071 \\
\hline stage & 1.434 & 0.870 & 2.363 & 0.157 \\
\hline grade & 1.222 & 0.878 & 1.702 & 0.235 \\
\hline T & 1.434 & 0.870 & 2.363 & 0.157 \\
\hline KPNA2 & 1.705 & 1.304 & 2.230 & 0.000
\end{tabular}

Tumor stage(III-IV).

\begin{tabular}{lllll}
\hline age & 0.999 & 0.980 & 1.019 & 0.945 \\
\hline gender & 1.185 & 0.651 & 2.159 & 0.579 \\
\hline stage & 2.075 & 0.639 & 6.740 & 0.225 \\
\hline grade & 1.131 & 0.754 & 1.698 & 0.552 \\
\hline T & 1.703 & 0.815 & 3.558 & 0.157 \\
\hline KPNA2 & 1.646 & 1.210 & 2.240 & 0.001
\end{tabular}

Table 2 


\begin{tabular}{|llll|}
\hline Clinical characteristic & Total (N) & Odds ratio in KPNA2 expression & P-Value \\
\hline Age(continuous) & 343 & $0.679 \llbracket 0.444-1.040 \rrbracket$ & 0.075 \\
\hline Stage(I vs II) & 253 & $2.080 \rrbracket 1.221-3.542 \rrbracket$ & 0.007 \\
\hline Stage(I vs III) & 254 & $2.342(1.372-3.999)$ & 0.002 \\
& & & \\
\hline Stage & & & \\
\hline (I-II vs III-IV) & 343 & $1.588(0.976-2.584)$ & 0.063 \\
& & & 0.001 \\
\hline Tumor status (T1 vs T2) & 257 & $1.956(1.157-3.308)$ & \\
\hline Tumor status (T1 vs T3) & 247 & $2.160(1.244-3.749)$ & 0.006 \\
\hline Tumor status (T1-T2 vs T3-T4) & 343 & $1.719(1.046-2.825)$ & 0.033 \\
\hline Grade (II vs III) & 286 & $1.852(1.150-2.985)$ & 0.011 \\
\hline Grade (I vs III) & 163 & $2.187(1.086-4.407)$ & 0.028 \\
\hline Grade (I-II VS III-IV) & 343 & $2.174(1.391-3.399)$ & \\
\hline
\end{tabular}

Table 3 


\begin{tabular}{|c|c|c|c|c|c|}
\hline \multirow[t]{3}{*}{ Description } & \multirow[t]{3}{*}{ Gene markers } & \multicolumn{4}{|l|}{ LIHC } \\
\hline & & \multicolumn{2}{|l|}{ TUMOR } & \multicolumn{2}{|l|}{ NORMAL } \\
\hline & & $\mathrm{R}$ & $P$ & $\mathrm{R}$ & $\mathrm{P}$ \\
\hline \multirow[t]{2}{*}{ CD8+ T cell } & CD8A & $0.282^{\star \star}$ & 0.000 & $0.339 *$ & 0.016 \\
\hline & CD8B & 0.284 ** & 0.000 & 0.236 & 0.100 \\
\hline \multirow[t]{2}{*}{ T cell (general) } & $\mathrm{CD} 2$ & 0.186 ** & 0.000 & 0.223 & 0.12 \\
\hline & CD3E & $0.163^{\star \star}$ & 0.002 & 0.25 & 0.081 \\
\hline \multirow[t]{2}{*}{ B cell } & CD19 & 0.072 & 0.166 & 0.081 & 0.574 \\
\hline & CD79A & 0.08 & 0.123 & 0.214 & 0.135 \\
\hline \multirow[t]{7}{*}{ Natural killer cell } & KIR2DL1 & 0.004 & 0.935 & $0.296 *$ & 0.037 \\
\hline & KIR2DL3 & 0.154 ** & 0.003 & 0.198 & 0.168 \\
\hline & KIR2DL4 & $0.205^{\star \star}$ & 0.000 & $0.535^{\star \star}$ & 0.000 \\
\hline & KIR3DL1 & 0.056 & 0.282 & $0.341^{*}$ & 0.015 \\
\hline & KIR3DL2 & 0.054 & 0.296 & 0.193 & 0.178 \\
\hline & KIR3DL3 & 0.016 & 0.754 & 0.193 & 0.18 \\
\hline & KIR2DS4 & 0.058 & 0.265 & 0.185 & 0.197 \\
\hline Neutrophils & CCR7 & 0.023 & 0.663 & $0.356^{*}$ & 0.011 \\
\hline Th1 & STAT4 & 0.037 & 0.475 & $0.283^{*}$ & 0.047 \\
\hline \multirow[t]{4}{*}{ Th2 } & GATA3 & 0.280 ** & 0.000 & $0.345^{\star}$ & 0.014 \\
\hline & STAT6 & -0.035 & 0.497 & $0.323^{*}$ & 0.022 \\
\hline & STAT5A & $0.143^{\star \star}$ & 0.005 & $0.307^{*}$ & 0.03 \\
\hline & IL13 & 0.055 & 0.292 & 0.172 & 0.233 \\
\hline Tfh & BCL6 & 0.056 & 0.284 & $0.507 * \star$ & 0.000 \\
\hline \multirow[t]{2}{*}{ Th17 } & STAT3 & $0.199 \star *$ & 0.000 & $0.442^{\star \star}$ & 0.001 \\
\hline & IL17A & -0.001 & 0.982 & 0.17 & 0.238 \\
\hline \multirow[t]{2}{*}{ T cell exhaustion } & CTLA4 & $0.282^{\star \star}$ & 0.000 & $0.311^{*}$ & 0.028 \\
\hline & LAG3 & $0.289 \star *$ & 0.000 & 0.208 & 0.148 \\
\hline \multirow[t]{2}{*}{ Mast cells } & TPSB2 & -0.064 & 0.214 & 0.083 & 0.564 \\
\hline & TPSAB1 & -0.066 & 0.201 & 0.173 & 0.231 \\
\hline
\end{tabular}




\begin{tabular}{lllll|} 
CPA3 & -0.03 & 0.563 & 0.196 & 0.173 \\
\hline MS4A2 & -0.042 & 0.423 & 0.096 & 0.509 \\
\hline HDC & $-0.119 *$ & 0.021 & $0.310 *$ & 0.029 \\
\hline
\end{tabular}

Figures
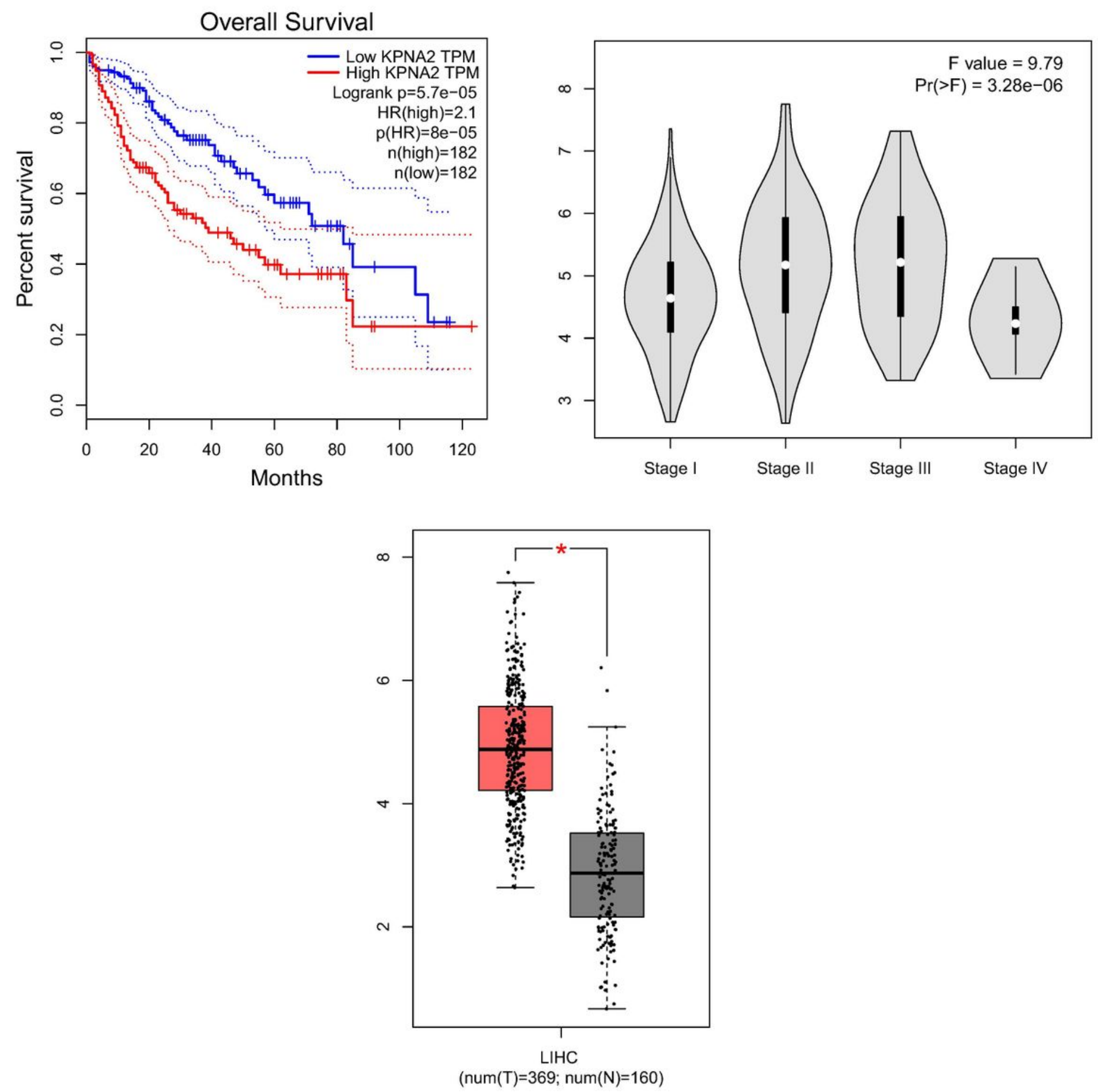

Figure 1 
Survival outcome and expression difference analyzed by GEPIA.

A. Increased KPNA2 expression is associated with bad outcome.

B. Differential expression of KPNA2 in different disease state (Tumor or Normal).

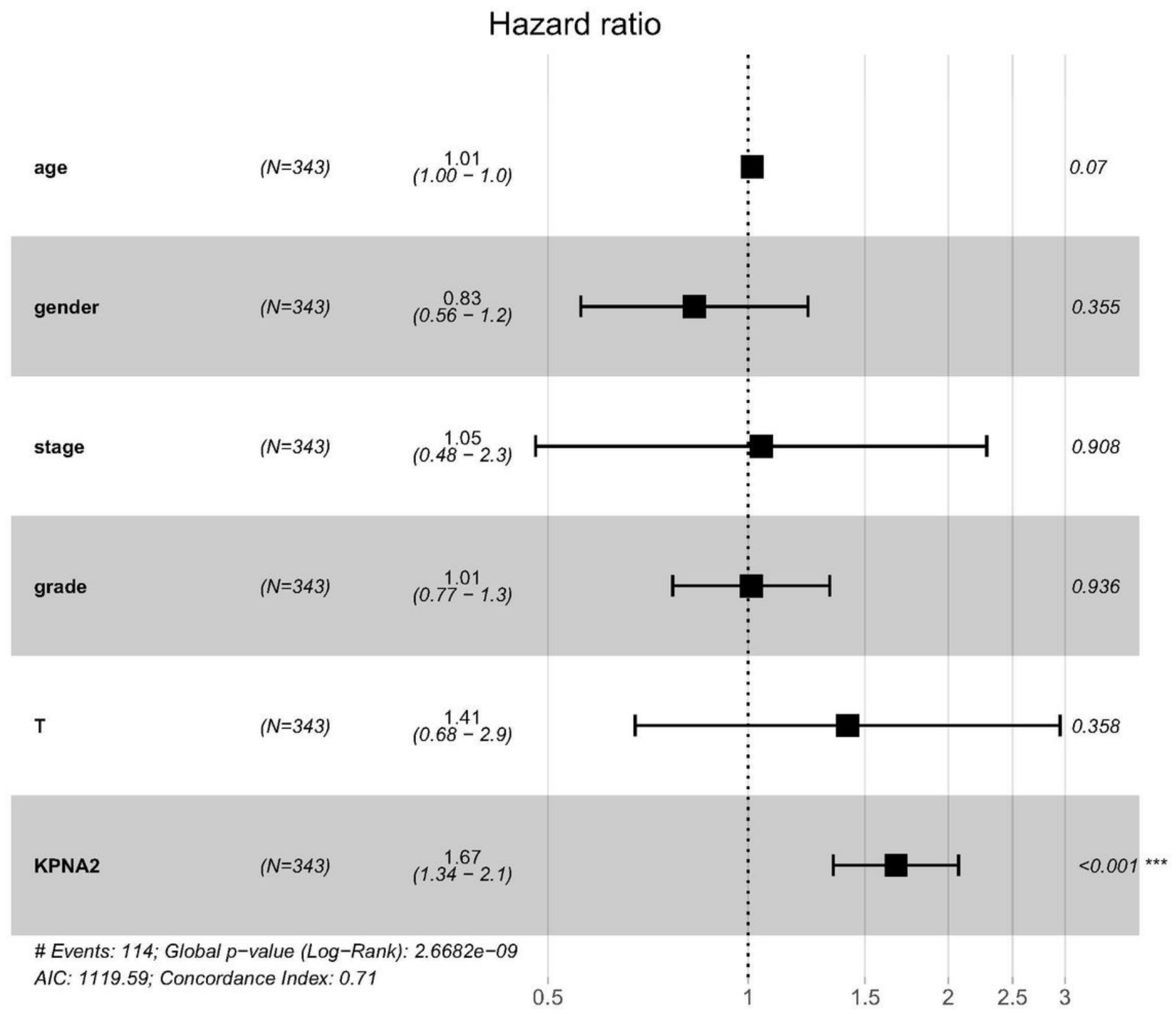

\section{Figure 2}

Multivariate Cox analysis of KPNA2 expression and other clinicopathological factors. //The downregulated KPNA2 expression is independent prognostic factors of favorable prognosis. 

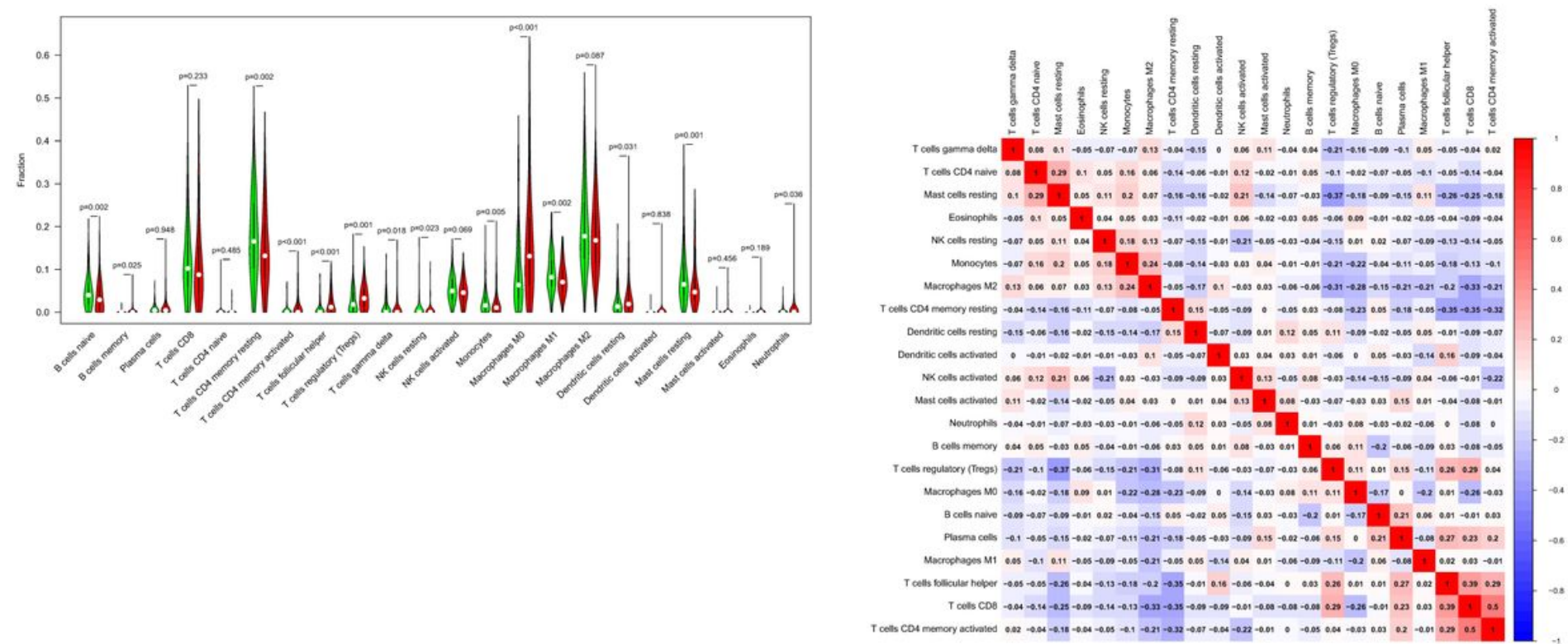

Figure 3

KPNA2-related immune infiltration alteration.

A. B cells memory $(p=0.002)$, T cells CD4 memory activated $(p<0.001)$, T cells follicular helper $(p<$ $0.001)$, Macrophages M0 ( $<0.001)$, Dendritic cells resting $(p=0.031)$, Neutrophils $(p=0.036)$ share a higher proportion in high expression group compared with low expression group. In contrast, the proportion of B cells naïve ( $p=0.002)$, T cells CD 4 memory resting $(p=0.002)$, NK cells resting $(p=$ $0.023)$, Monocytes $(p=0.005)$, Macrophages M1 $(p=0.002)$, Mast cells resting $(p=0.001)$, are apparently lower.

B. The proportions of different TIICs subpopulations were weakly to moderately correlated. 

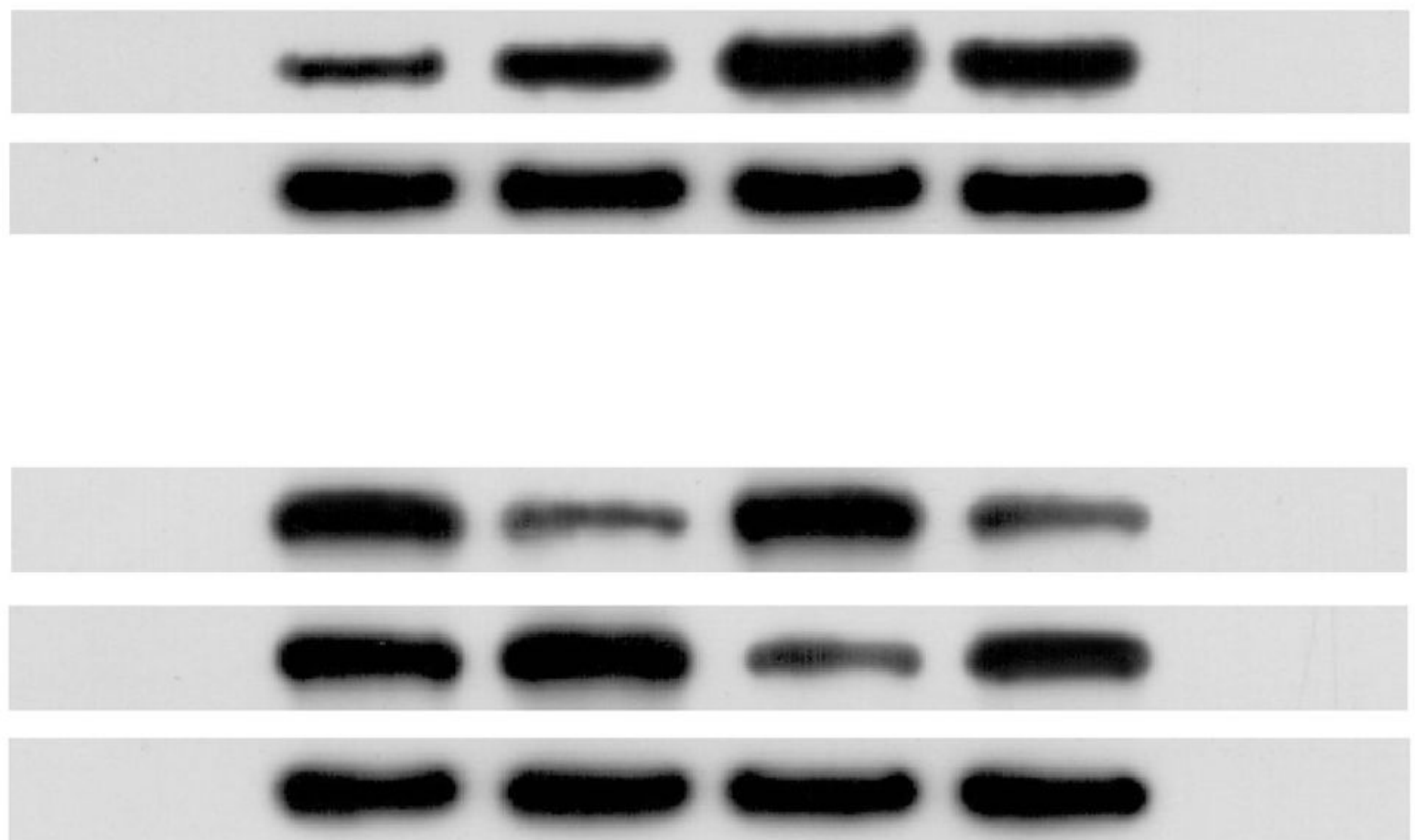

Figure 4

Figure legend not available with this version. 